\title{
An Account of Quantum Interference from a Hydrodynamical Perspective
}

\author{
A. S. Sanz \\ Instituto de Física Fundamental, Consejo Superior de Investigaciones Científicas \\ Serrano 123, 28006 - Madrid, SPAIN
}

In the last years, Bohmian mechanics [1,2] has passed from being an almost unknown curiosity within the foundations of quantum mechanics to a well-known (and increasingly accepted) theory from the computational [3] and interpretational [4,5] viewpoint. This arises from a very nice appealing feature: within this approach quantum processes and phenomena can be understood on similar grounds as classical ones, i.e., in terms of the motion displayed (in configuration space) by a swarm of trajectories representing the evolution of a quantum flow. This idea can be traced back to the hydrodynamical picture of quantum mechanics proposed by Madelung [6], which underwent a rebirth in the 1970s through the works of Bialynicki-Birula and coworkers [7] or Hirschfelder and coworkers [8].

In this communication, different distinctive properties associated with quantum interference, such as interference, tunnelling or nonlocality, will be analyzed and discussed from the hydrodynamic perspective provided by Bohmian mechanics [3,4,9-11]. As it is shown, when attention is primarily paid to the quantum probability current density instead of the probability density, very interesting and challenging quantum properties arise. Though they might seem to be an artefact arising only in Bohmian mechanics, actually they were "hidden" within the conventional way we usually look at quantum mechanics -to some extent this can be compared to the nonlocality property of this theory [12], which is always present, but emerges very strikingly in experiments like the very well-known EPRB one [13]. Also, far from a mere academic exercise, another purpose here is to stress the potential interest and power of Bohmian mechanics at both the fundamental and the applied levels. At a fundamental level, due to the insight it provides to analyze all our preconceived notions of quantum processes and phenomena; at an applied level, because of the direct implications in fields such as atom and BEC interferometry or quantum information, for example. In this regard, another key point of this communication will be the insight Bohmian trajectories provide to understand concepts such as decoherence or quantum erasure -in the case of loss of fringe visibility with light, the electromagnetic approach analogous to Bohmian mechanics will be considered [11].

\section{References}

[1] Bohm: Phys. Rev. 85, 166, 184 (1952).

[2] Holland: The Quantum Theory of Motion (Cambridge University Press, 1993).

[3] Wyatt: Quantum Dynamics with Trajectories (Springer, 2006).

[4] Sanz, Borondo, S. Miret-Artés: J. Phys.: Condens. Matter 14, 6109 (2002).

[5] Sanz, Miret-Artés: in Quantum Dynamics of Complex Molecular Systems, Micha and Burghardt (eds.), Springer Series in Chemical Physics, 83 (Springer, 2006), p. 343-368.

[6] Madelung, Z. Physik 40, 322 (1926).

[7] Bialynicki-Birula, Bialynicka-Birula: Phys. Rev. D 3, 2410 (1971);

Bialynicki-Birula, Cieplak, Kaminski: Theory of Quanta (Oxford University Press, 1992).

[8] C. Hirschfelder

[9] Sanz, Miret-Artés: J. Phys. A 41, 435303 (2008).

[10] Chou, Sanz, Miret-Artés, Wyatt: Phys. Rev. Lett. 102, 250401 (2009).

[11] Sanz, Davidović, Božić, Miret-Artés: Ann. Phys. 325, 763 (2010).

[12] Bell: Physics 1, 195 (1964); Rev. Mod. Phys. 38, 447 (1966).

[13] Aspect, Grangier, Roger: Phys. Rev. Lett. 49, 91 (1982). 